\title{
Braucht es wirklich ein Institut für Praxisinformatik?
}

Christian Peier

Geschäftsführer IPI

Korrespondenz:

Dr. med. Christian Peier

IPI - Institut für Praxisinformatik c/o Hausärzte Schweiz

Geschäftsstelle

Effingerstrasse 54

Postfach 6052

CH-3001 Bern

c.peier[at]praxisinformatik.ch

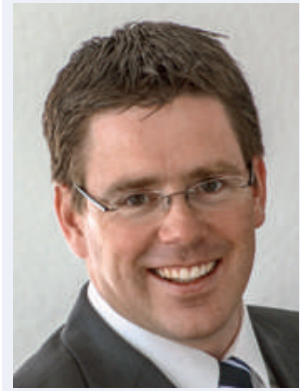

Unser IPI-

Geschäftsführer: Dr. med. Christian Peier

Wie im vorangegangenen Artikel zur Mandatserteilung für die Praxisinformatik durch die FMH an das Institut für Praxisinformatik (IPI) erwähnt, hat das IPI per 1.3.2014 einen vollamtlichen Geschäftsführer berufen. Wir freuen uns sehr, mit Dr. med. Christian Peier (Jg. 1973) einen äusserst kompetenten, engagierten und mit der Materie bestens vertrauten Experten gefunden zu haben. Dr. med. Christian Peier ist mit einer Ärztin verheiratet und hat zwei schulpflichtige Söhne. Er hat nach seinem Medizinstudium an der Universität Bern früh neben dem medizinischen auch sein IT-Interesse entdeckt und dies zu seinem Beruf gemacht. Nach 2-jähriger Tätigkeit in einer medizintechnischen Firma baute er 2003 zusammen mit einem Informatiker eine führende Firma für Softwarelösungen und e-KG in den Arztpraxen auf, wo er während 10 Jahren als

\section{Braucht es wirklich ein Institut für Praxisinformatik?}

Eine berechtigte Frage! Bevor ich jedoch darauf eingehe, möchte ich eine Geschichte erzählen. Sie ist zusammen mit einem guten Freund und Branchenkenner entstanden, während wir uns Gedanken machten, wie die Softwarehäuser anstelle der Software (im gleichen Stil) Autos verkaufen würden:

Eine Familie geht in eine Garage und möchte ein neues Auto kaufen. Sie möchten ein zuverlässiges Fahrzeug mit gewissem Komfort - keinen Ferrari; aber etwas Gehobeneres als ein Dacia. Sicherheit und Qualität sind ihnen wichtig.

Der Fachhändler erfragt ihre Bedürfnisse und Wünsche, um ihnen das optimale Fahrzeug verkaufen zu können. Er zeigt ihnen verschiedene Bilder und zählt mögliche Funktionalitäten auf. Es fallen u. a. Stichworte wie «Lane Assist», «Bremsassistent mit Notbremsung», «ECO PRO Route» und «Abstandsregeltempomat». Auch von «Stop \& Go-Funktion» und «Park Assist», von «Kessy» sowie von «BAS Plus mit Kreuzungsassistent» ist die Rede. Einige Begriffe hat das Paar noch nie gehört oder kennt sie nicht wirklich. Und so stellt sich die Frage, ob sie
Mitglied der Geschäftsleitung tätig, für das Projektmanagement und als Leiter CustomerCare für die Praxis-Beratungen zuständig war.

Als Mitglied der Gruppe eHealth (eCH) bis 2009, Vertreter der IT-Branche im IFAS-Messe Beirat und vor allem als Mitglied des Vorstandes des Verbandes Schweizerischer Fachhäuser für Medizininformatik (VSFM, Verband der Softwarefirmen) bis 2013, kennt er die Branche bestens und ist mit allen wichtigen Playern im eHealth-Bereich vernetzt. Mit dieser seltenen, aber hervorragenden Kombination von ärztlichem Fachwissen und gründlichen IT-Kenntnissen ist Christian Peier wahrlich prädestiniert für den Job als Geschäftsführer des IPI. Das Kernteam des IPI hat soeben eine intensive Klausurtagung hinter sich und sich bereits zu einem äusserst engagierten, «verschworenen» Team gefunden. Wir wünschen Christian Peier viel Erfolg und grosse Genugtuung in seiner spannenden Aufgabe.

Im folgenden Artikel skizziert der Geschäftsführer des IPI seine Vorstellungen und Visionen.

Gerhard Schilling, Präsident IPI

denn all die neuen und tollen Funktionen wirklich brauchen. Im Dialog mit dem geduldigen Fachberater werden schliesslich die Kriterien für den neuen Wagen festgelegt.

Gemeinsam begeben sie sich in den Ausstellungsbereich und besichtigen ein entsprechendes Objekt. Beim Anblick bemerkt der Vater verunsichert: «Grundsätzlich gefällt mir das Auto. Es hat aber keine Räder! Ist es denn fahrtauglich?» «Aber sicher!» entgegnet der Verkäufer, «natürlich werden wir ihnen diese vor der Auslieferung gern als Extras montieren - mit neuen 18-Zoll-Felgen und neuen Pneus.» Der Fachmann führt die Familie zum Heck des Autos, öffnet den Kofferraumdeckel und schwärmt: «Sehen Sie das grosszügige Raumangebot und das ausgeklügelte Gepäckhalterungssystem!» «Aber der Kofferraum ist zu klein für unseren Kinderwagen», meint da die Mutter. Der Verkäufer lächelt und meint gelassen: «Brauchen Sie den wirklich? Meine Frau und ich brauchen auch keinen. Ansonsten, verstauen Sie ihn auf dem Rücksitz. Dort hat es Platz und ich bin sicher, Sie werden sich rasch daran gewöhnen. Ich habe bereits viele Kunden, die das so machen.» 


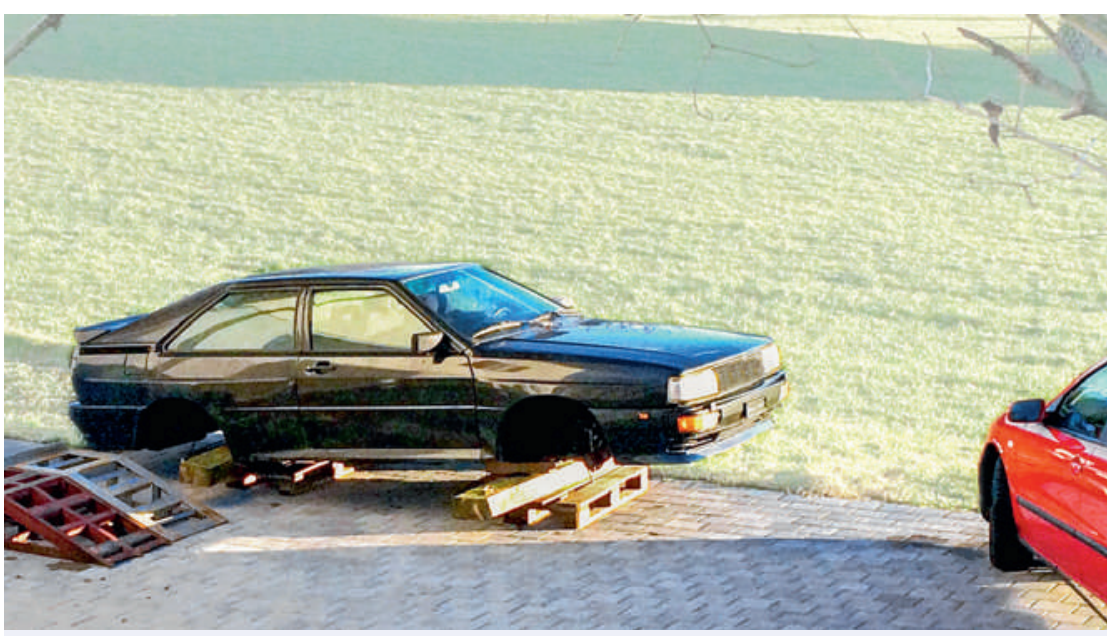

Sieht das Wunschauto wirklich so aus? alleine da und sind nicht bzw. ungenügend in die bestehende Software integriert. Daneben fehlen Standards und Regeln für einen Austausch von Daten. Als Arzt stellt sich mir die Frage nach der Wirksamkeit, der Zweckmässigkeit und der Wirtschaftlichkeit von Funktionen. Wie beim Tarifkatalog sollte diese Fragestellung auch in der eHealth angewendet werden. Die Frage nach dem Nutzen für Patienten und Leistungserbringer soll dabei im Vordergrund stehen.

\section{Gemeinsam ans Ziel}

Meine langjährige Erfahrung hat gezeigt, dass die Entwicklung neuer Funktionen nur mit dem Verständnis des ärztlichen Schaffens und der Praxisprozesse möglich ist. Es sind die Ärztinnen und Ärzte, die täglich mit diesen Systemen arbeiten und sie wissen am besten, was es braucht.

Es kann nicht sein, dass neue, geniale Tools entwickelt werden, die im Alltag weder nützlich noch zweckmässig sind. Und es kann nicht sein, dass überall aufwendige eigenständige Projekte umgesetzt werden, die dann mangels Nachfrage wieder verschwinden. Wir brauchen einen Rahmen - ein gemeinsames Ziel. Und es braucht ein Miteinander.

Mit meiner Arbeit am Institut für Praxisinformatik setze ich mich dafür ein, dass die Arbeit mit IT einfacher und effizienter wird. Die Arbeit soll Spass machen und die Leistungserbringer sollen sich vermehrt auf ihre Patienten konzentrieren können. Daneben sollen sie von genialen Tools unterstützt werden und ein besseres Verständnis für die IT haben. Der Wechsel von Papier- auf elektronische Krankengeschichten steht vielen noch bevor. Es handelt sich um einen Wechsel, der in keiner Hinsicht unterschätzt werden darf. Mit dem IPI kann ich aus Erfahrung wertvolle Hilfestellung leisten.

\section{Einladung zur Mitarbeit}

Ich sehe es als meine Aufgabe, Ihre Bedürfnisse strukturiert $\mathrm{zu}$ formulieren und sie sowohl der ITBranche als auch der Politik vorzulegen und zu vertreten. Das schaffe ich nicht alleine. Ich bin auf Ihre Hilfe angewiesen. Deshalb lade ich Sie ein, sich aktiv an der Gestaltung der Zukunft zu beteiligen. Geben Sie mir Ihr Feedback! Teilen Sie mir Ihre Wünsche und Anregungen, aber auch Ihre Sorgen, Fragen und Ärger mit. Wir vom IPI werden diese Anregungen sichten und bündeln und am runden Tisch mit den Softwarefirmen besprechen. Wenn alle Beteiligten Ärzte, Patienten, IT-Industrie und die Politik - gemeinsam am gleichen Strick ziehen, so entwickeln wir eines der besten eHealth-Systeme der Welt. 